\title{
SAÚDE GERAL E SINTOMAS DEPRESSIVOS EM UNIVERSITÁRIOS
} GENERAL HEALTH AND DEPRESSIVE SYMPTOMS IN UNDERGRADUATE

Recibido: 14 de Diciembre del 2011 | Aceptado: 10 de Agosto del 2012

\author{
ADRIANA MUNHOZ CARNEIRO ${ }_{1}$; MAKILIM NUNES BAPTISTA 2 \\ (UNIVERSIDADE SÃO FRANCISCO, Itatiba, SP, Brasil).
}

\begin{abstract}
RESUMO
0 presente estudo teve como objetivo principal buscar evidências de validade baseada na relação com outras variáveis para a Escala de Depressão- EDEP com o Questionário de Saúde Geral -QSG-60, avaliando também se os grupos diferenciariam quanto ao sexo, faixa etária, nível sócio econômico ou histórico de doença mental na família. Participaram deste estudo 98 universitários, com idade média de 25,8 anos $(D P=7,72)$. Correlações moderadas entre os instrumentos foram encontradas, indicando que a presença de transtornos mentais comuns encontra-se relacionada ao aumento de sintomas depressivos. Ainda, sexo, faixa etária, classe sócio econômica e histórico de doença mental na família não diferenciaram os escores de saúde geral e sintomas depressivos.
\end{abstract}

PALAVRAS-CHAVE: Depressão; Saúde mental; Testagem psicológica; Validade.

\section{ABSTRACT}

This study aimed to search evidences of validity, searching evidences of validity based on the relation to other variables to Escala de Depressão -EDEP with the Questionário de Saúde Geral QSG -60, assessing differences in groups according to gender, age group, socio-economic class, and history of family mental disease. Took part of this study 98 undergraduated students, mean age 25,8 (SD=7,72). Moderate correlations between instruments were found, indicating that the presence of common mental disorders is related to increased depressive symptoms. Furthermore, sex, age group, socioeconomic class and history of mental disease did not differentiate scores of general health and depression symptoms.

KEY WORDS: Depression, Mental health, Psychological testing, Validity.

1. Psicólogo. Discente do curso de Pós-Graduação Stricło Sensu - Mestrado em Psicologia da Universidade São Francisco, Itatiba, SP; bolsista CAPES. 2. Psicólogo. Doutor pelo Departamento de Psiquiatria da Escola Paulista de Medicina (UNIFESP); Docente do Programa de Pós-Graduação Strico-Sensu em Psicologia da Universidade São Francisco - Itatiba, SP; bolsista Produtividade pelo CNPq.

Endereço do autor responsável:

Programa de Pós Graduação Strictu-Sensu em Psicologia

Comissão de Pós Graduação. Av Alexandre Rodrigues Barbosa, 45 - Centro, CEP 13251-900, Itatiba-SP. Telefone: (11) 4534-8040

Email: makilim.baptista@saofrancisco.edu.br. 
INTRODUÇÃO

Quando se estuda a respeito da saúde geral, é possível identificar diferentes formas pelas quais a mesma já foi citada, visto que seu conceito perpassa por constantes modificações, tendo influências culturais, sociais, políticas e econômicas (Sciliar, 2007). Conforme o mesmo autor argumenta, a definição do que poderia ser considerado enquanto saúde possui explicações em que os conceitos de cura e normalidade se transformam, e juntamente, o que poderia ser considerado como cura, ou como doença. De acordo com Sá Junior (2004) os conceitos de saúde mental se modificam a partir do momento em que se verificam transtornos mentais em diversas populações, demonstrando que ter algum tipo de transtorno mental não é um castigo divino, como se acreditava na antiguidade, mas sim, fruto de fatores genéticos, sociais e culturais.

Atualmente, a World Health Organization (2000) tenta conceituar o que poderia ser considerado como saúde, definindo-a como um "estado de perfeito bem-estar físico, mental e social", evidenciando assim a saúde mental como uma das partes que integram a saúde geral (Segre \& Ferraz, 1997). Nesse sentido, deve-se ressaltar que essa definição ainda é longe de ser completa, já que pode-se inferir a saúde como ausência de doenças, assim como questionar 0 que seria um estado de perfeição, devendo mais estudos discutirem sobre este aspecto.

Apesar do conceito de saúde abranger mais do que a ausência de doença mental, 0 estudo sobre quais seriam os principais gatihos para 0 desenvolvimento de transtornos mentais é de suma importância para o avanço de pesquisas na área de prevenção da saúde, e já foi foco de estudo de vários pesquisadores. Alguns deles, os quais este trabalho se pauta, justificam 0 desenvolvimento de transtornos mentais pela forma pela qual o sujeito interpreta os eventos, indicando assim, que a vulnerabilidade a eventos estressores também estará associado ao transtorno mental (Beck, Rush, Shaw \& Emery, 1982; Freeman, Simon, Beutler \& Arkowitz, 1989; Guic, Bilbao \& Bertim, 2002; Vindel, Salguero, Wood, Dongil \& Latorre, 2012).

Vindel e colaboradores (2012), por exemplo, demonstram em seu trabalho de revisão de literatura que a depressão se mostra como um transtorno que causa importantes danos na qualidade de vida física e psicológica do sujeito. Apontam que a depressão necessita ser corretamente diagnosticada e tratada, não apenas para redução dos custos, mas também para poder propiciar uma melhor qualidade de vida ao sujeito. Assim, torna-se importante observar alguns dados provenientes de outras pesquisas a respeito da relação entre os construtos avaliados, uma vez que apesar de ser um tema escasso na literatura, se mostra como um dado necessário para facilitar desenvolvimento de instrumentos de prevenção psicológica e tratamentos de promoção. A seguir, seguem alguns estudos que demonstram esta relação entre os construtos avaliados.

Cerchiari, Caetano e Faccenda (2005) avaliaram a prevalência de Transtornos Mentais Menores em universitários dos cursos de Computação $(n=73)$, Direito $(n=159)$, Letras $(n=69)$ e Enfermagem $(n=257)$, com idades entre 19 e 24 anos, sendo $71 \%(n=167)$ do sexo feminino. Os participantes responderam ao QSG- 60 e a um questionário sócio demográfico, cujos resultados indicaram que os fatores mais pontuados na amostra foram desconfiança no próprio desempenho, distúrbios psicossomáticos e estresse psíquico, indicando estas variáveis como as que mais poderiam predispor o sujeito a desenvolver transtornos mentais, indiferente da idade ou sexo. Outro estudo sobre saúde geral foi realizado com estudantes do curso de Turismo $(n=236)$, sendo $69 \%(n=162)$ do sexo feminino, com idades entre 15 a 21 
anos, aplicando o QSG-60. Os resultados foram separados por sexo, e indicou que as mulheres possuem maior ausência de saúde mental, sendo esta diferença de média estatisticamente significativa (Cerchiari, Garcia, \& Facenda, 2009). Similar ao estudo anterior, os fatores mais pontuados em ambos os sexos foram estresse psíquico e desconfiança no próprio desempenho, o que vem a demonstrar que estes sujeitos provavelmente avaliam a si e ao mundo de forma mais negativa, sentindose assim, mais vulnerável.

Souza, Baptista e Alves (2008) estudaram a relação entre percepção de suporte familiar e saúde geral, mediante a aplicação do Inventário de Percepção de Suporte Familiar -IPSF e do QSG. Dos 520 universitários, $329(63,3 \%)$ eram do sexo feminino, com idade média de 25,04 anos $(D P=6,29)$. Os resultados indicaram correlações negativas do QSG-60 com o IPSF, em que quanto menor a saúde mental, pior a percepção de suporte familiar do sujeito. Ainda quanto ao QSG as mulheres pontuaram mais do que os homens nos fatores Stress Psíquico, Desconfiança no Próprio Desempenho, Distúrbios do sono, Distúrbios Psicossomáticos e Severidade de Doença Mental. Os autores também correlacionaram os resultados do QSG com a classificação sócio econômica, encontrando relações significativas no fator Stress Psíquico e Distúrbios Psicossomáticos, ou seja, quanto melhor o nível econômico dos participantes, menor estresse e distúrbios psicossomáticos.

Em um estudo mais recente, Gujardo e Cadena (2012) encontraram como resultados que $90 \%$ dos estudantes se queixaram de insônia e distúrbios do sono, assim como de ansiedade (65\%) e estresse emocional e físico (90\%). Participaram da pesquisa 134 universitários, que responderam a um questionário com 10 itens que visava atender 0 objetivo principal da pesquisa, que era pontuar as alterações de saúde que eram mais frequentes em estudantes do $6^{\circ}$. Semestre de enfermagem. Isso fez com que os autores concluíssem que o grupo avaliado não possuía condições de saúde adequada para as exigências requeridas durante a graduação, e também na necessidade de se realizar condutas preventivas nessa população, já que esta é uma população que raramente se tem trabalhos de promoção de saúde.

Estudos específicos sobre as relações de saúde mental e depressão abrem um tópico que interessará cada vez mais, tendo em vista a grande incidência de sintomas depressivos na população. Entende-se que depressão se configura como o transtorno de humor mais comum mundialmente, sendo que vários fatores contribuem para sua incidência (APA, 2002; Bahls, 2000; $\mathrm{WHO}, 2006)$. Este aumento, que muitas vezes se classifica enquanto uma epidemia silenciosa, e faz com que sejam necessárias formas de se prevenir o agravamento destes sintomas, principalmente, considerando os gastos que esta incide, sejam pessoais, sociais ou econômicos, muitas vezes o paciente, por não saber dos sintomas do transtorno, recorre primeiro ao serviço médico que trata os sintomas físicos da doença mental, não havendo diagnóstico do transtorno mental, acarretando em um tempo maior de sofrimento (Morgan \& et al., 2008; Watkins \& et al., 2009).

Kinder et al. (2008) verificaram que a depressão apareceu como associada ao risco de morte após aproximadamente dois anos para pacientes que já tiveram alguma história de depressão ou estresse pós traumático em cuidados primários. 0 estudo se baseou em 35715 pacientes que completaram ao Health Checklist. Destes, $6876 \quad(19,3 \%)$ tinham histórico de depressão, $748(2,1 \%)$ de estresse pós traumático e $3762(10,5 \%)$ ambos. A idade dos participantes variou entre 19 a 98 anos. Ainda em relação aos resultados, é interessante apontar que ter apenas 
estresse pós traumático não teve relações com o aumento do risco de morte, indicando que a vulnerabilidade ao desenvolvimento dos sintomas depressivos pode estar relacionado ao desenvolvimento de comorbidades, já que os depressivos podem se colocar em comportamentos de saúde mais nocivos, que os levam a aumentar a propensão a desenvolvê-las.

Izadinia, Amiri, Jahromi, Hamidi (2010) investigaram as relações entre resiliência, estresse, saúde mental, depressão e ansiedade com pensamentos suicidas em 265 universitários, com idades entre 22 e 32 anos. Os participantes responderam ao General Health Questionnaire- QHQ, Beck Depression Inventory - BDI, Beck Anxiety Inventory - BAI, Beck Scale for Suicide Inventory- BSI e Connor and Davidson's Resiliency scale. Os resultados demonstraram correlações significativas entre resiliência e pensamento suicida, sendo que quanto maiores os pensamentos suicidas, menor a resiliência, tal como correlações significativas entre estresse diário, depressão, saúde mental e ansiedade, indicando que quanto maiores os índices de transtornos mentais $\mathrm{e}$ estresse, maior a tendência do sujeito ter pensamentos suicidas. Complementarmente, realizou-se uma análise de regressão para verificar qual 0 papel preditivo destas variáveis no pensamento suicida, verificando que a depressão tem a maior parcela na previsão de pensamentos suicidas, seguido pela ansiedade, saúde mental, resiliência e estresse diário.

Em relação ao achado pelos autores, vale considerar que a questão do sexo pode influenciar diretamente nestes resultados, já que é possível verificar que os homens manifestam sintomas depressivos de forma diferente das mulheres. De forma geral, a literatura evidencia que os homens optam por formas mais externalizantes, tendo com maior freqüência irritabilidade e raiva, uso de álcool, procura por medicações, chegando até a tentativa de suicídio, ao passo que as mulheres tendem a ter comportamentos mais internalizantes, como o embotamento afetivo, a tristeza e a desesperança (APA, 2005; Kornstein, 1997). Assim sendo, alguns estudos apontam que os homens tenderiam a expressar seus sentimentos com menor frequencia por meio de palavras, o que funcionaria como um fator que pode influenciar nos resultados dos testes, ou ainda gerar uma descompensação na detecção de casos masculinos de depressão na detecção de casos masculinos com depressão (APA, 2002; Kornstein, 1997; Kposowa \& McElvain, 2006).

Ainda, pode-se verificar que as pesquisas apontam a relação entre sintomas depressivos com muitas outras variáveis, tais como neuroticismo, baixo suporte social, elevados níveis de estresse, distúrbios do sono e desesperança, o que demonstra a crescente preocupação da comunidade científica em identificar variáveis relacionadas com a mesma e em como de prevení-la, atrelada a validação e criação de escalas de detecção de sintomas depressivos (Baptista \& Carneiro, 2008; Baptista, Carneiro \& Sisto, 2010; Borine, 2009; Gomes, 2009; Gutman, 2010, Panzarella \& et al., 2006; Santos \& Kassouf, 2006). Cabe considerar que os resultados das pesquisas podem indicar o quanto é possível a utilização de testes psicológicos, trazendo evidências psicométricas importantes para que o pesquisador e/ou clínico se armem de métodos válidos e fidedignos em suas práticas (AERA, APA \& NCME, 1999). Assim sendo, há a importância em se pensar também na relevância de se construir instrumentos considerando a cultura e a sociedade na qual o sujeito está inserido ao se optar pelo uso de determinado instrumento que mensure a depressão (Gotlib \& Hammen, 2009; Pasquali, 1999). 
A lista de instrumentos psicológicos construídos no país e validados ainda é pequena quando comparada ao número de comportamentos psicológicos que necessitam ser mensurados, em que 0 Sistema de Avaliação de Testes Psicológicos -SATEPSI (CFP, 2012), traz uma lista atualmente com de pouco mais de 100 testes aprovados para a utilização, entretanto, existem apenas dois instrumentos disponíveis para avaliação de depressão, elucidando a necessidade de estudos na área e de aprimoramento da EDEP. Nesse sentido, visando contribuir para o aumento do número de testes psicológicos na área, o objetivo deste estudo foi 0 de encontrar evidências de validade baseada na relação com outras variáveis para a Escala de Depressão EDEP, correlacionando ao Questionário de Saúde Geral -QSG -60, uma vez que a literatura aponta a necessidade de averiguar o impacto dos sintomas depressivos na saúde geral. Secundariamente, serão avaliadas diferenças entre sexo, estado civil, faixa etária, classe sócio econômica e histórico de doença mental familiar dos respondentes quanto aos escores obtidos em cada teste.

\section{MÉTODO}

\section{Participantes}

Participaram do estudo 98 estudantes, escolhidos por conveniência, de uma universidade particular do estado de Rondônia, sendo 56 (57,1\%) do sexo feminino, dos cursos de Direito $(n=46$, correspondendo a $46,9 \%$ da amostra) e Psicologia $(n=15$, correspondendo a $15,3 \%$ dos participantes). A idade dos participantes variou de 17 a 49 anos ( $M=25,8 ; D P=7,72)$. Quanto ao estado civil, 60 (61,2\%) eram solteiros, 26 ( $n=26,5 \%)$ casados, $5(5,1 \%)$ desquitados/divorciados, 2 (2\%) recasados e $4(4,1 \%)$ não responderam a questão.

Perguntou-se aos participantes também sobre a presença de histórico de depressão na família ou pessoal. Destes, 40 (40,8\%) responderam que algum membro familiar já havia sido diagnosticado com depressão maior. Do total que identificaram o familiar com depressão, $13(13,3 \%)$ responderam ser a mãe, oito $(8,2 \%)$ pai e tia, cinco $(5,1 \%)$ a irmã, dois (2\%) avó e dois (2\%) respondeu ser mais de um familiar com depressão e um $(1 \%)$ indicou ter mais de dois familiares com depressão. Dos participantes, 61 $(62,2 \%)$ afirmaram nunca ter passado por avaliação psicológica ou psiquiátrica. Os participantes que responderam que sim, oito $(8,2 \%)$ indicaram ter sido diagnosticados com depressão e dois $(2 \%)$ responderam outros transtornos, sendo eles anorexia e fobia, além do que, 95 participantes $(96,9 \%)$ indicaram não estar passando por tratamento para depressão e três $(3,1 \%)$ não responderam a questão.

\section{Instrumentos}

Questionário de identificação: questionário elaborado com questões abertas (idade, curso, membro com transtorno mental na família) e fechadas (sexo, estado civil). Juntamente ao questionário, incluiu-se 0 critério de classificação econômica da Associação Brasileira de Empresas de Pesquisa (ABEP, 2010).

Questionário de Saúde Geral de GoldbergQSG: (Pasquali, Gouveia, Almeida \& Ramos, 1996): este questionário foi construído por Goldberg (1972) com 0 intuito de identificar a severidade de distúrbios psiquiátricos menores. A seleção dos itens foi realizada de forma que não descrevesse transtorno de personalidade para que fosse possível de se aplicar em uma população geral. Permite avaliar a severidade de distúrbios psiquiátricos nãopsicóticos e servir como meio de identificação de casos potenciais dos mesmos na população geral não clínica (faixa etária de 20 a 70 anos). A versão principal inclui 60 itens, sendo populares outras versões, com 30, 20, 12, 28 e 30 itens, devendo ser respondidas em uma 
escala Likert de quatro pontos. Possui cinco fatores, F1 Stress Psíquico, F2 Desejo de Morte, F3 Falta de Confiança na própria capacidade de desempenho, F4 Distúrbios do sono e F5 Distúrbios Psicossomáticos, além do fator Total.

Escala de Depressão - EDEP (Baptista, 2009): escala construída conforme indicadores do DSM-IV-TR (APA, 2002), CID10 (OMS, 1993), Terapia Cognitiva (Beck \& et al., 1982) e Teoria Comportamental (Ferster, 1977) da depressão. Na construção da escala, foram elaboradas 150 sentenças, distribuídas em 75 itens e após análise de conteúdo, os itens foram reduzidos a 32 , contendo 18 descritores, divididos em somáticos, vegetativos, sociais e cognitivos. Esta é uma versão preliminar da escala final, sendo que os respondentes possuem para cada item duas alternativas de resposta, devendo responder com base nas últimas semanas, em uma escala tipo Likert de três pontos. Foram realizadas análises por meio da Teoria de Resposta ao Item- TRI e da estrutura interna da versão com 32 itens. Estes indicaram que os índices de infit e outfit foram adequados, sendo os valores médios de infit de 1,06 (DP= 0,42) para as pessoas e $1,02(\mathrm{DP}=0,24)$ para os itens e os de outfits com média de $1,02(D P=0,48)$ para as pessoas e 1,02 ( $\mathrm{DP}=0,30)$ para os itens, tendo apenas dois itens com valores acima do recomendado, demonstrando que 0 teste se encontrava dentro dos parâmetros e com boa distribuição. Quanto ao índice das pessoas, esses foram dentro do esperado $(1,00)$, sendo a precisão do teste de 0,92 para o item e de 0,90 para as pessoas. Atualmente, essa escala é denominada de Escala Baptista de Depressão- versão adulto -EBADEP-A e possui 45 itens em sua versão final.

\section{Procedimentos}

Após a aprovação do projeto pelo Comitê de Ética e pela instituição de ensino, os questionários foram apresentados aos universitários. Após o consentimento dos mesmos por meio do Termo de Consentimento Livre e Esclarecido -TCLE, procedeu-se a aplicação dos instrumentos, tendo uma duração média de 30 minutos. A aplicação foi coletiva, e a ordem dos instrumentos foi 0 questionário de identificação, seguido da EDEP e QSG, não tendo mudanças em sua ordem de aplicação.

\section{RESULTADOS}

Primeiramente, para as análises estatísticas inferenciais, realizou-se uma análise correlacional entre O QSG e a EDEP. Para essa análise, utilizou-se a correlação de Pearson, de forma a verificar a direção das mesmas, tal como pode ser visualizado na Tabela 1.

TABELA 1.

Correlação de Pearson (r) da EDEP com os fatores do QSG.

\begin{tabular}{ccccccc}
\hline & $\begin{array}{c}\text { Stress } \\
\text { Psiquico }\end{array}$ & $\begin{array}{c}\text { Desejo da } \\
\text { morte }\end{array}$ & $\begin{array}{c}\text { Desconfiança no } \\
\text { próprio desempenho }\end{array}$ & $\begin{array}{c}\text { Distúrbios do } \\
\text { Sono }\end{array}$ & $\begin{array}{c}\text { Distúrbios } \\
\text { psicossomáticos }\end{array}$ & Total \\
\hline EDEP & $0,70\left(^{* *}\right)$ & $0,55\left(^{* *}\right)$ & $0,66\left({ }^{* *}\right)$ & $0,57\left({ }^{* *}\right)$ & $0,57\left({ }^{* *}\right)$ & $0,74\left({ }^{* *}\right)$ \\
\hline
\end{tabular}

** Correlação a nível de 0.01 .

Tal como demonstra a Tabela 1 , a relação com o fator total foi significativa e de alta magnitude, indicando que quanto maior a sintomatologia depressiva, pior a saúde geral, ou seja, maior o comprometimento de sua saúde. Ainda, a análise correlacional considerando cada um dos fatores do QSG demonstra que estas foram de magnitude moderada nos fatores Desejo da morte,
Distúrbios psicossomáticos e do Sono e alta nos fatores estresse psíquico e Desconfiança no próprio desempenho. Ambos os fatores que apontaram correlações altas possuem conteúdos referentes a desesperança, identificação de sentimentos como tristeza, raiva e incapacidade, itens que também são contemplados na EDEP. 
Sequencialmente, para que fossem possíveis as análises de diferenças de média, verificou-se quais foram as médias dos sexos no que se refere a pontuação dos instrumentos. Estas informações constam na Tabela 2.

TABELA 2.

Descrição de pontuação média e diferença de média dos participantes (n=98) em relação a EDEP e QSG.

\begin{tabular}{lccccc}
\hline & Sexo & Média & DP & $t$ & $p$ \\
\hline \multirow{2}{*}{ EDEP } & masculino & 47,40 & 8,95 & $-0,12$ & 0,905 \\
& feminino & 47,64 & 10,31 & & \\
QSG Stress psíquico & masculino & 25,62 & 7,60 & $-0,64$ & 0,522 \\
& feminino & 26,68 & 8,43 & & \\
QSG Desejo de morte & masculino & 9,73 & 3,50 & $-0,71$ & 0,481 \\
QSG Desconfiança no próprio & feminino & 10,23 & 3,37 & & \\
desempenho & masculino & 33,58 & 7,20 & $-1,46$ & 0,148 \\
QSG Distúrbios do sono & feminino & 36,05 & 9,10 & & \\
\multirow{2}{*}{ QSG Distúrbios psicosomáticos } & masculino & 10,07 & 3,90 & $-0,81$ & 0,418 \\
QSG Total & feminino & 10,75 & 4,24 & & \\
& masculino & 17,19 & 4,45 & $-2,09$ & 0,039 \\
\hline
\end{tabular}

Conforme aponta a Tabela 2, a pontuação dos sexos na EDEP foi similar, sendo que as mulheres tiveram uma média um pouco acima da masculina, e o mesmo se deu no QSG Total e em seus fatores, sendo que a única diferença de média estatisticamente significativa se deu no fator Distúrbios psicossomáticos do QSG, indicando que as mulheres tendem a relatar com maior freqüência estes distúrbios do que os homens. Analisou -se também se existiriam diferenças de média entre os grupos no que concerne ao curso, idade, estado civil e nível sócio econômico, entretanto, não foram encontradas diferenças de média entre os grupos nos escores dos instrumentos.

Para analisar diferenças quanto a idade, dividiu-se a mesma em quartis, sendo estas classificadas por até 20 anos $(n=32$, correspondendo a $32,7 \%)$, de 21 a $23(n=16$, com $16,3 \%)$, de 24 a 29 ( $n=20$, equivalente a $20,4 \%)$ e mais que 30 anos $(n=21$, correspondendo a $21,4 \%$ ). Apesar da divisão em faixas etárias, não foram encontradas diferenças significativas, indicando que os sintomas depressivos e a qualidade de saúde geral independem da idade nesta amostra. No que concerne ao estado civil e nível sócio econômico, também não foram encontradas diferenças significativas estatisticamente, indicando que os sintomas depressivos e saúde geral independem destas variáveis.

$\mathrm{Na}$ sequência, verificou-se se os escores dos participantes que assinalaram ter algum familiar diagnosticado com doença mental seriam maiores do que os que não possuem, e se esta diferença seria significante. $O$ teste $t$ demonstrou que apesar das médias dos respondentes serem maiores $(10,12)$ nos que tem algum parente com doença mental dos que não possuem $(9,49)$, estas não foram estatisticamente significativas $(t=-0,11 ; p=$ 0,91).

\section{DISCUSSÃO}

Pouco se tem na literatura a respeito de estudos analisando a relação entre saúde geral e sintomas depressivos. Como verificado, a EDEP (atual EBADEP-A) traz alguns estudos que contribuem com evidências de validade para a mesma, tal como o presente estudo, em que correlações de moderada a alta magnitude entre os 
instrumentos apontam evidências de validade baseada na relação com outras variáveis relacionadas. Assim, quanto maiores os sintomas de estresse, desejo de morte, desconfiança em si, alterações de sono e sintomas psicossomáticos, maiores também os sintomas depressivos.

A construção de instrumentos conforme a cultura e a sociedade na qual será aplicado é de suma importância, e é discutida por outros autores também (Gotlib \& Hammen, 2009; Pasquali, 1999). Bem como Gotlib e Hammen (2009) colocaram, é importante também levar em consideração a finalidade para a qual o instrumento será aplicado, visto que a depressão é multifatorial, e pode ser mensurada em diversas formas, ora focando mais aspectos cognitivos, por exemplo, ora fatores somáticos ou afetivos. Deve-se considerar que um instrumento pode ter todas as evidências de validade e precisão possíveis, mas ainda assim, não ser um bom instrumento para o contexto desejado, por isso, deve se atentar a finalidade a qual se opta por aplicar determinado instrumento (Urbina, 2007).

$\mathrm{Na}$ presente amostra, 0 instrumento mostrou propriedades psicométricas adequadas, e contribui para a formação de evidências de validade para a versão final da escala. Prieto e Muñiz (2000) classificam que os instrumentos que medem construtos equivalentes, com magnitudes acima de 0.60 devem ser interpretados como excelentes, e confirmam o esperado, uma vez que os itens do QSG, apesar de não serem equivalentes, associam-se com os da EDEP no que concerne ao desejo de morte, estresse, entre outros itens somáticos e afetivos.

Conforme aponta Derogatis e Lynn (1999) são várias as vantagens de um instrumento de screening, e uma delas, se dá principalmente por este poder auxiliar na detecção do potencial do sujeito desenvolver ou de agravar os sintomas dos transtornos mais graves. Considerando que a EDEP é um instrumento construído para funcionar como screening, e como tal, mensurando sintomas depressivos, e não diagnosticando depressão, deve-se atentar também para as desvantagens relacionadas ao uso de quaisquer instrumentos de rastreamento, ou seja, a probabilidade aumentada da mesma em mensurar falsos positivos. Ainda, vale ressaltar que não existe uma análise que indique quais são os possíveis pontos de corte do instrumento, para que seja possível diferenciar casos possíveis de não casos, o que faz com que os resultados sejam vistos com ressalvas. Desta forma, como relatam Derogartis e Lynn (1999) cada situação de screening deve ser realizada considerando o contexto ao qual se aplica o teste, 0 que indica novamente a importância do contexto cultural na validação e uso de uma escala já apontada por Pasquali (1999).

Em se tratando da correlação encontrada entre os instrumentos e a suas magnitudes, torna-se relevante considerar que este corrobora com o pressuposto de outros autores, tal como Morgan et al. (2008), Ozabaci (2010), Sobocki et al. (2007), Vindel et al. (2012) e Watkins et al. (2009), os quais trouxeram em suas pesquisas a importância de se avaliar as relações entre 0 desenvolvimento de sintomas depressivos com a saúde mental. Complementarmente, 0 presente estudo também vai ao encontro dos mesmos por indicar relações significativas entre estes dois construtos, indicando assim, que aqueles que melhor avaliavam sua saúde geral se percebem com menos sintomas depressivos, independente de sexo ou idade, talvez até porque muitos dos sintomas nas duas escalas se sobrepõem.

A correlação encontrada no que concerne a dimensão estresse psíquico e depressão é congruente ao apresentado, respectivamente, pelas teorias que comentam acerca do desenvolvimento de transtornos mentais, tal como a de Beck et al. (1982), os quais já atentavam que o 
aumento de vulnerabilidade aos estressores tende a gerar uma maior propensão a desenvolver transtornos mentais, tal como a depressão. Este resultado também corrobora as conclusões obtidas por Izadinia et al. (2010), que também verificaram que os que avaliavam melhor suas condições apresentavam maior resiliência e menores índices de pensamentos suicidas.

O estudo de Izadinia et al. (2010) ainda apontou que a depressão tendia a contribuir com grande parte nos pensamentos suicidas. No presente estudo, o desejo de morte, dimensão do QSG, estava correlacionado com a EDEP, indicando que quanto maiores os escores nesta dimensão, maiores os sintomas depressivos, corroborando com 0 indicado por estes autores. A dimensão desconfiança no próprio desempenho do QSG com a EDEP também aponta que a percepção negativista sobre si e o mundo tende a aumentar os sintomas depressivos como um todo, e corrobora com 0 proposto nos manuais e literatura da área, que já afirma que 0 sujeito com depressão tende a ter este viés quanto a sua percepção de ambiente e de si (APA, 2002; APA, 2005; Izadinia \& et al., 2010; Kornstein, 1997; Panzarella \& et al., 2006).

A partir dos resultados encontrados, também vale comentar sobre a correlação entre distúrbios do sono e distúrbios psicossomáticos com a EDEP. A respeito destes resultados, ainda existe uma carência de pesquisas sobre a relação de distúrbios psicossomáticos e sintomatologia depressiva, podendo-se afirmar, entretanto, que a depressão vem aparecendo como aquela que está relacionada ao desenvolvimento de sintomas físicos, tal como trouxe o estudo de Kinder et al. (2008). Gotlib e Hammem (2009) apontam uma série de doenças relacionadas com transtornos depressivos, como por exemplos, as doenças cardíacas, o qual demonstra que 0 desencadeamento de transtornos emocionais parecem estar relacionados com problemas físicos nos sujeitos.

Assim como em outros estudos (Cerchiari \& et al., 2005; Cerchiari \& et al., 2009) não foram encontradas diferenças entre os cursos ou entre os sexos nos resultados do QSG, independente do número de participantes. Este resultado traz divergências com o encontrado por Souza et al. (2008), em que as mulheres apresentaram maior desconfiança no próprio desempenho, distúrbios psicossomáticos e do sono, ao mesmo tempo em que corrobora com outros achados, como por exemplo, as diferenças significativas entre os sexos, demonstrando que as mulheres apresentaram maiores indicadores de transtornos psiquiátricos, sendo que no estudo a amostra também eram de universitários.

Já quanto a EDEP, no que se refere ao sexo, o estudo vai de encontro ao de Santos e Kassouf (2006) que também foi realizado no país e indicou que as mulheres percebiam mais sintomas depressivos do que os homens. Os achados no presente estudo podem indicar que o tipo e o número da amostra podem interferir diretamente nos escores encontrados entre os sexos. No que tange aos outros resultados, idade, estado civil e nível sócio econômico não tiveram diferenças de média estatisticamente significativas. Achados semelhantes foram realizados em Cerchiari et al. (2005) e Cerchiari et al. (2009), demonstrando que a idade não interferia na saúde mental, em que o mesmo se deu no QSG. Na EDEP, cabe ressaltar que no presente estudo a idade dos participantes concentrou-se nos 25 anos ( $\mathrm{DP}=7,72$ ), desta forma, não é possível determinar se 0 aumento substancial da idade poderia apontar diferenças de média, como por exemplo, no estudo de Santos e Kassouff (2006), que observaram em sua amostra que 0 aumento da idade incidia em maiores riscos de surgimento da depressão, sendo 
este aumentado em 0,00162 por ano de idade nos homens, 0,0432 nas mulheres.

Quanto aos resultados encontrados em relação ao estado civil, o estudo corrobora com os achados de Cerchiari et al. (2005) e Cerchiari et al. (2009), que também não tiveram diferenças influenciadas pelo estado civil. Como ressalvas a estes resultados, vale lembrar que a maior parte dos respondentes indicou ser solteira nesta pesquisa, tal como na amostra dos autores supramencionados, assim, não se pode considerar que a condição de estar solteiro possa ser favorável ou não a saúde mental, necessitando mais estudos a respeito.

Referente à classe sócio econômica, mais de $50 \%$ dos participantes foram agrupados na classe $B$, e os resultados não indicaram diferença significativa entre as classes. Ao contrário destes resultados Cerchiari et al. (2009) e Souza et al. (2008) encontraram diferenças de média, em que quanto maior o nível econômico, maior a saúde geral medida pelo QSG. Outro resultado que traz diferenças de manuais e literatura (APA, 2002; Laffer \& Vallada Filho, 1999; OMS, 1993) é o fator genético, no caso, avaliado pela questão que perguntava sobre um parente direto com sintomas de depressão, demonstrando que esta variável não influenciou os escores dos participantes.

Para finalizar, ressalta-se algumas das limitações do estudo que são a amostra, sugerindo também, que em estudos posteriores preconizem grupos critério (sujeitos com depressão ou outros transtornos mentais já diagnosticados) e 0 número da amostra, que por ter sido de conveniência, foi diminuta. Sugere-se em próximos estudos intercalar a ordem dos testes para controlar 0 efeito fadiga $e$ analisar também eventos de vida juntamente com estas variáveis, tendo em vista que estas são potenciais para detectar problemas de saúde mental e/ou depressão.

\section{REFERÊNCIAS}

American Psychiatric Association (2002). Manual Diagnóstico e Estatístico de transtornos mentais: DSM IV TR. Tradução de Cláudia Dorneles. $\left(4^{\mathrm{a}}\right.$ ed.). Porto alegre: Artmed.

American Psychological Association (2005). Men: a different depression [on line]. Disponível em: http://www.apa.org/research/action/me n.aspx. Acesso em: 13 jul. 2011.

American Educational Research Association [AERA], American Psychological Association [APA], Nacional Council on Measurement in Education [NCME] (1999). Standards for Educational and Psychological Testing. Washington, DC: American Educational Research Association.

Associação Brasileira de Empresas de Pesquisa [ABEP] (2010). Critério de Classificação Econômica Brasil. Disponível em: http://remonato.pro.br/documents/Crit\% C3\%A9rio\%20de \%20Classifica\%C3\%A 7\%C3\%A30\%20Econ\%C3\%B4mica $\% 2$ OBrasil\%20-\%20ABEP\%202010.pdf. Acesso em: 1.1.11.

Bahls, S-C. (2000). Uma visão geral sobre a doença. Revista InterAÇÃO, 4, 61-73.

Baptista, M. N. (2009). Escala de Depressão- EDEP. Relatório Técnico não Publicado. Programa de Pós graduação Strictu Senso em Psicologia. Universidade São Francisco.

Baptista, M. N., \& Carneiro, A. M. (2008, maio) Relações entre depressão, percepção do suporte familiar e desesperança. Trabalho apresentado no XIV Encontro de

Iniciação Científica, Universidade São Francisco, Bragança Paulista, São Paulo.

Baptista, M. N., Carneiro, A. M., \& Sisto, F. F. (2010). Estudos Psicométricos de Escalas de Depressão (EDEP e BDI) e o Inventário de Percepção de Suporte 
Familiar - IPSF. Psicologia em Pesquisa, 4(1):65-73.

Beck, T. A., Rush, J. A., Shaw, F. B., \& Emery, G. (1982). Terapia cognitiva da Depressão. Rio de Janeiro: Zahar.

Borine. B. (2009). Escala de Depressão (EDEP) e Bateria Fatorial de personalidade: Evidências de Validade. Dissertação de Mestrado, Programa de Pós- Graduação Stricto Sensu em Psicologia, Universidade São Francisco, Itatiba.

Cerchiari, E. A., Garcia, D. S., \& Facenda, O. (2009). Estudo do perfil sociodemográfico e da prevalência de Transtornos mentais menores em estudantes de turismo da UEMS/ Unidade de Dourados. Turismo em Análise 20(3), 563-567.

Cerchiari, E. A. N., Caetano, D., \& Faccenda, O. (2005). Prevalência de transtornos mentais menores em estudantes universitários. Estudos de Psicologia, 10(3), 413-420.

Conselho Federal de Psicologia -CFP (2012). Sistema de Avaliação de Testes Psicológicos- SATEPSI. Conselho. Disponível em: http://www.pol.org.br/satepsi/. Acesso em: 22. jun. 2010.

Derogatis, L. S., \& Lynn, L. L. (1999). Psychological tests in screening for Psycriatric Disorder. In: M. E. Mariush. The use of psychological testing for treatment planning and outcomes assessment (2a. ed.). New Jersey: Lawrence Erlbaum Associates.

Ferster, C. B., Culbertson, S., \& Boren, C. P. (1977). Princípios do comportamento. (Maria Ignez Rocha e Silva, Maria Alice de Campos Rodrigues e Maria Benedita Lima Pardo, Trad.). São Paulo: Hucitec.

Freeman, A., Simon, K., Beutler, L. E., \& Arkowitz, H. (1989). Comprehensive Handbook of Cognitive Therapy. New York: Plenun Press.

Gomes, J. O. (2009). Escala de Depressão (EDEP) e medidas de Atenção e Transtornos de sono. Dissertação de
Mestrado, Programa de PósGraduação Stricto Sensu em Psicologia, Universidade São Francisco, Itatiba, 96p.

Gotlib, I. H., \& Hammen, C. L. (2009). Handbook of depression. 2a. Ed. New York: The Guilford Press.

Gouveia, V. V., Chaves, S. S. S., Oliveira, I. C. P., Dias, M. R., Gouveia, R. S. V., \& Andrade, P. R. (2003). A utilização do QSG-12 na população geral: estudo de sua validade de construto. Psicologia teoria e pesquisa 19(3), 241-248.

Guic, E., Bilbao, M. A., \& Bertin, C. (2002). Estrés Laboral y salud en una muestra de ejecutivos chilenos. Revista médica de Chile, 130(10), 1101-1112.

Gujardo, L. C., \& Cadena, D. M. G. (2012). Alteraciones de salud en estudiantes de licenciatura en enfermería. Revista Electrónica de Investigación en Enfermería, 1(1), 16-21.

Izadinia, N., Amiri, M., Jahromi, R. G., \& Hamidi, S. (2010). A study of relationship between suicidal ideas, depression, anxiety, resiliency, daily stresses and mental health among Tehran university students. Procedia Social and Behavioral Sciences, 5, 1515-1519.

Kinder, L. S., Bradley, K. A., Katon, W. J., Ludman, E., McDonell, M. B., \& Bryson, C. L. (2008). Depression, posttraumatic stress disorder, and mortality. Psychossomatic Medicine, 70, 20-26.

Kornstein, S. G. (1997). Gender Differences in Depression: Implications for Treatment. Journal of Clinical Psychiatry, 58(supl15), 12-18.

Kposowa, A. J., \& McElvain, J. P. (2006). Gender, plale and method of suicide. Society of Psychiatry Epidemiology, 41, 435-443.

Laffer, B., \& Vallada Filho, H. P. (1999). Genética e fisiopatologia dos transtornos depressivos. Revista Brasileira de Psiquiatria, 21(1). Disponível em: http://www.scielo.br/scielo.php?pid=S1 
51644461999000500004\&script=sci_ar ttext. Acesso em: 21 jun. 2011.

Morgan, R. O., Byrne, M. M., Hughes, R. B., Petersen, N. J., Taylor, H. B. Robinson-Whelen, S., Hasche, J. C., \& Nosek, M. A. (2008). Do secondary conditions explain the relationship between depression and health care cost in women with psysical disabilities? Archives of Physical Medicine and Rehabilitation, 89, 1880 1886.

Organização Mundial da Saúde -OMS (1993). Classificação Internacional de Doenças - CID10. Porto Alegre: Artes Médicas.

Ozabaci, N. (2010). Quality of life as a predictor of depression. Procedia Social na Behavioral Sciences, 2, 2457- 2462.

Panzarella, C., Alloy, L. B., \& Whitehouse, W. G. (2006). Expanded Hopelessness Theory of Depression: On the mechanisms by which social support protects against depression. Cognitive Therapy Res., 30, 307-333.

Pasquali, L. (1999). Instrumentos psicológicos: manual prático de elaboração. Brasília: LabPAM; IBAPP. 306p.

Pasquali, L., Gouveia, V. V., Andriola, W. B., Miranda, F. J., \& Ramos, A. L. M. (1994). Questionário de saúde geral de Goldberg (QSG): adaptação brasileira. Psicologia teoria e pesquisa, 10(3), 421-437.

Prieto, G., \& Muniz, G. (2000). Un modelo para evaluar la calidad de los tests utilizados en España. Disponível em: http://www.cop.es/vernumero.asp?id=4 1. Acesso em: 03 nov. 2010.

Sá Junior, L. S. de M. (2004). Desconstruindo a definição de saúde. Jornal do Conselho Federal de Medicina. Disponível em: http://www.dis.unifesp.br/pg/Def-

Saude.pdf. Acesso em: 05. jan. 2011.

Santos, M. J., \& Kassouf, A. L. (2006). Uma estimativa econométrica do retorno da educação para a saúde mental dos brasileiros: escolaridade versus depressão. Acesso em: 18. mai. 2008. Disponível em: http://www.cepea.esalq.usp.br/pdf/educ acao_depressao.pdf.

Sciliar, M. (2007). História do Conceito de Saúde. Revista de Saúde Coletiva, 17(1), 29-41.

Segre, M., \& Ferraz, F. C. (1997). O conceito de saúde. Revista de Saúde Pública, 31(5), 538-542.

Sobocki, P., Ekman, M., Agren, H., Krakau, I., Runeson, B., Martensson, B., \& Jonsson, B. (2007). Health- related quality of life measured with EQ- 5D in Patients Treated for Depression in Primary Care. International Society of Pharmacoeconomics and Outcomes Research -ISPOR, 10(2), 153-160.

Souza, M. S. de, Baptista, M. N., \& Alves, G. A. S. (2008). Suporte familiar e saúde mental: evidência de validade baseada na relação entre variáveis. Aletheia, 28,32-44. [on line]. Disponível em: [<http://pepsic.bvsalud.org/scielo.p $\mathrm{hp}$ ? script $=$ sci_arttext\&pid=S141303942008000200005\&lng=pt\&nrm =iso>. Acesso em: 13 jul. 2011.

Urbina, S. (2007). Fundamentos da Testagem Psicológica. Porto Alegre: Artmed.

Vindel, A. C., Salguero, J. M., Wood, C. M., Dangil, E., \& Latorre, J. M. (2012). La depresión en atención primaria: prevalencia, diagnóstico y tratamiento. Papeles del Psicologo, 33(1), 2-11.

Watkins, K. E., Burnam, M. A., Orlando, M., Escarce, J. J., Huskamp, H. A., \& Goldman, H. H. (2009). The health value and cost of care for major depression. International Society for Pharmacoeconomics and Outcomes Research, 65-72.

World Health Organization - WHO (2006). Conquering Depression. Mental Health and Substance Abuse. Recuperado em: 10 abr. 2008. Disponível em http://www.searo.who.int. 
World Health Organization [WHO] (2000). WHO initiative on Depression in Public Health. On Line. Recuperado em 30 de Abril, 2008, de http://www.who.int. 